\title{
Comparison of sensitivity of psychophysical and electrophysiological measures of scotopic thresholds in the vicinity of the blind spot'
}

H. H. PAYNe 2

U. S. NAVY ELECTRONICS LABORATORY, SAN DIEGO, CALIFORNIA

A section of the blind spot was mapped by obtaining detection and averaged EEG threshold measurements using three stimulus light intensities. It was found that averaged EEG and detection measures of the blind spot were equally sensitive when the two highest stimulus intensities were used, although more observations of the stimulus were required with averaged EEG. Detection thresholds were superior to averaged EEG measures with the dimmest stimulus.

It has been established by a number of workers (see a review by Rietveld (1963)) that the visually evoked cortical potential varies as a function of the intensity of the stimulus. Tepas and Armington (1962) found latency changes in the first negative wave of the averaged EEG and complicated amplitude changes depending on the size and intensity of the test stimulus. Cobb and Dawson (1960) found all waves of the evoked potential to decrease with decreasing stimulus intensity.

Practical applications of the sensitivity of the evoked potential to stimulus intensity have been found. Copehaver and Beinhocker (1963) described the use of "electroperimetry" as a supplement to ophthalmological perimetry.

The purpose of this study was to map the sensitivity of the retina with a scotopic stimulus in the neighborhood of the blind spot using averaged evoked cortical potentials and detection thresholds.

\section{Subjects}

\section{METHOD}

The author, 28 years of age with no known visual defects, served as the single $S$ in this experiment. $\mathrm{S}$ used only his left eye.

\section{Apparafus}

$S$ was equipped with a red fixation light mounted on a calibrated slide. A 5 min. stimulus light could be imaged at selected positions within and to the side of the blind spot of S's left eye. Figure 1 gives the approximate trajectory that the stimulus followed through the blind spot.

A device which regulated the probability of the occurrence of the stimulus light was set so the light appeared with probability one-haif.

The evoked potentials were recorded on an offner Dynagraph and fed into a TMC model 300C computer of average transients. The results were plotted on an $\mathrm{X}-\mathrm{Y}$ plotter.
The stimulus light was generated by a Grass PS2 photo-stimulator set on intensity setting I8 (manufacturer's specificiation-' 1500,000 peak candles measured at $10 \mathrm{in}$. from the face plate'). The $5 \mathrm{~min}$. stimulus apperture was centered on the reflector of the photostimulator: a .003 gauge acetate matte diffusing screen covered the apperture. Three intensities of the stimulating light were effected by placing $4.0,4.2$, and $4.4 \mathrm{log}$ neutral density Wratten filters in front of the face plate of the photostimulator.

With a dark adapted eye, the brightest stimulus $(\log 4.0)$ could not be seen with the fovea.

\section{Procedure}

$\mathrm{S}$ dark preadapted for $20 \mathrm{~min}$. before entering the shielded test room.

In obtaining the detection thresholds $\mathrm{S}$ positioned the fixation light. He then depressed a foot pedal; 1 sec. later the light appeared or did not appear. $S$ recorded whether he saw the stimulus; he then obtained information as to whether the stimulus appeared and recorded this. The stimulus light both appeared and did not appear at least 20 times each; that is, $S$ had at least 40 trials.

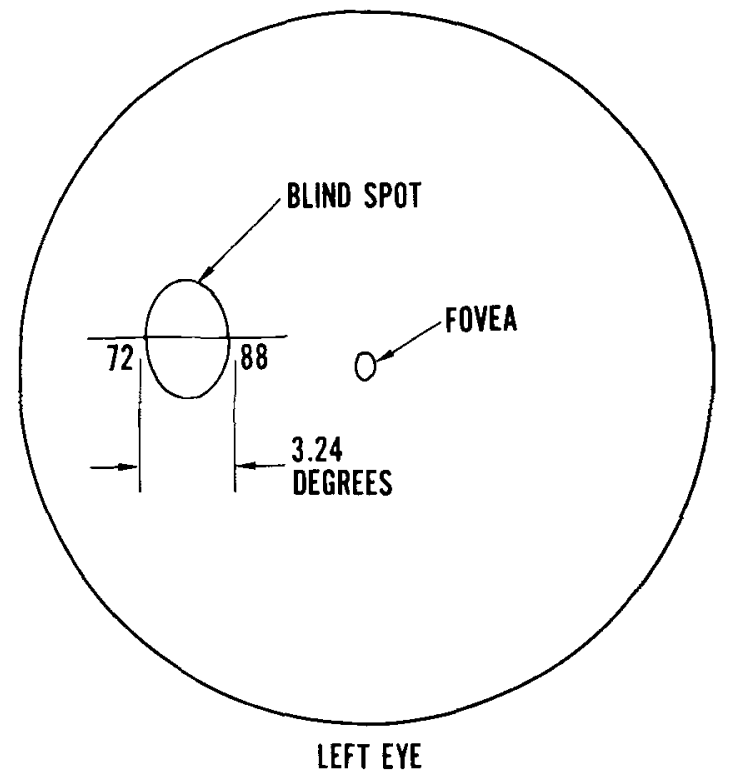

Fig. 1. Map of S's left eye. The line drawn through the blind spot indicates where a $5 \mathrm{~min}$. stimulus was projected. The numbers 72 and 88 show the orientation of an arbitrary scale. 

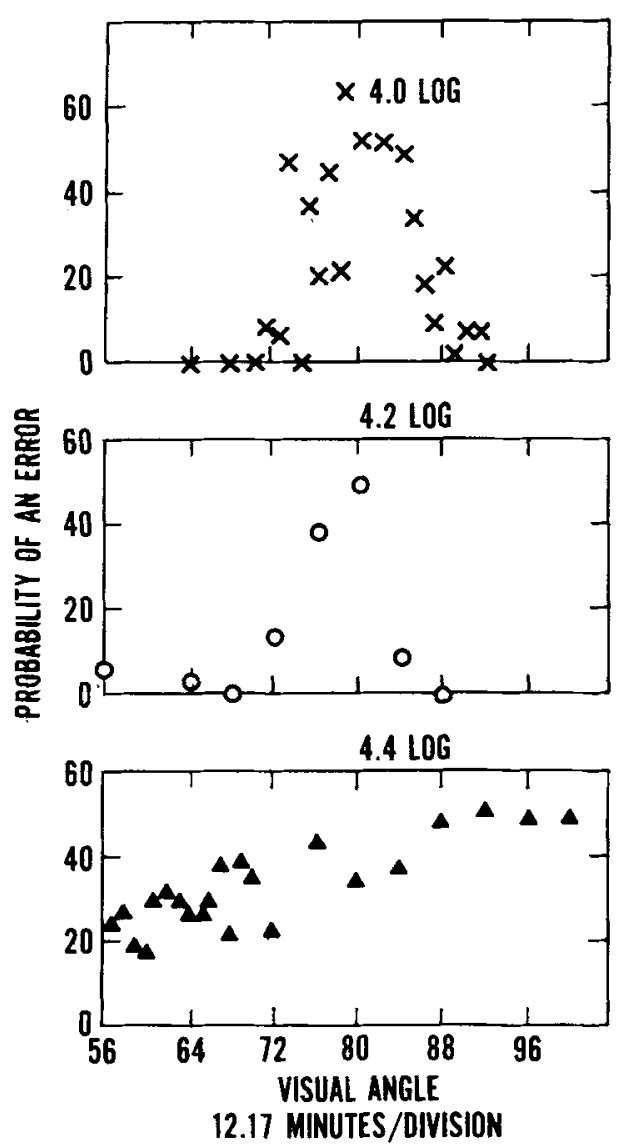

Fig. 2. Observed probability of an error (decimal point omitted) (S did not see-stimulus present or saw-stimulus not present) as a function of retinal location for three stimulus intensities: $4.0 \mathrm{Iog}$ is brightest and 4.4 is the dimmest. The abscissa units represent an arbitrary scale. $12.17 \mathrm{~min}$./division gives the conversion factor for determining visual angle.

Detection thresholds at 22 locations for intensity $\log 4.2$, and 22 locations for intensity $\log 4.4$ (the dimmest) were taken in random order within each intensity setting.

An electrode was placed 1 in. above and to right of S's inion. A ground electrode was attached to S's right ear.

$S$ positioned the fixation light. While fixating $S$ depressed a foot switch, held his breath, and remained as motionless as possible. One sec. later the stimulus light flashed. The EEG was recorded for 500 msec. after the flash of the light. S repeated this process 200 times; then average evoked potential was plotted.

Eleven positions at intensity $\log 4.0,13$ at $\log 4.2$ and 8 at $\log 4.4$ were investigated in random order, both with respect to retinal location and intensity. This design was then replicated in random order.

\section{RESULTS}

The observed probability of $S$ making an error (i.e., either saying the stimulus appeared when it
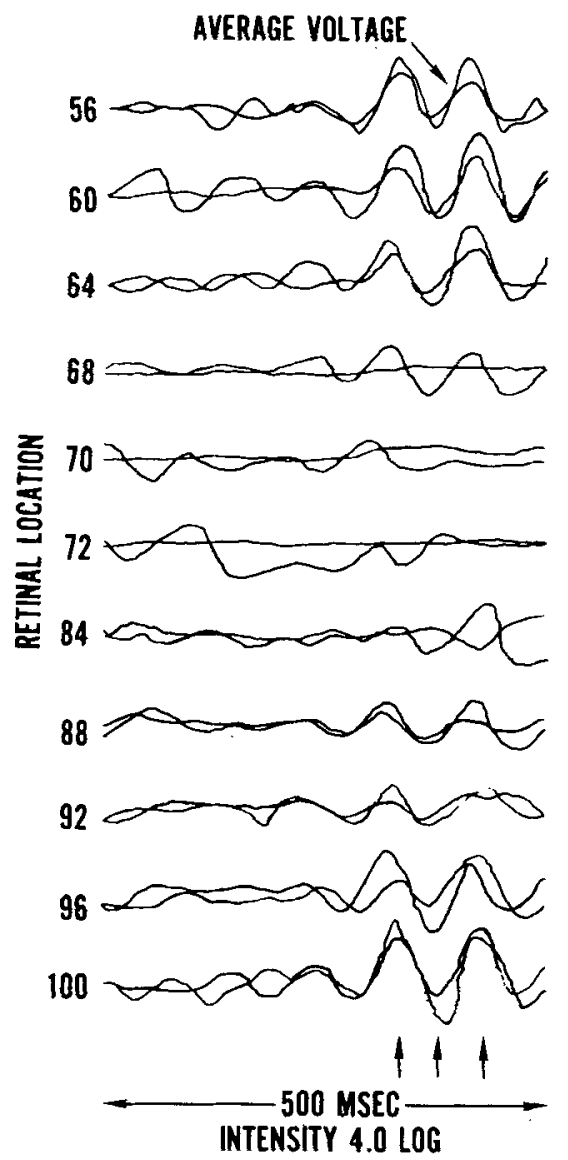

Fig. 3. Average voltage over a $500 \mathrm{msec}$. interval to the brightest stimulus light (intensity $\log 4.0$ ) as a function of retinal location. The three arrows (at bottom) are located at 325,375 , and $425 \mathrm{msec}$. and indicate the two peaks and one trough which are used to compute an amplitude measurement.

did not or saying it did not appear when it appeared) as a function of retinal location is plotted for three stimulus intensities in Fig. 2.

Figure 3 gives an example of the evoked potential for the $4.0 \mathrm{log}$ (highest) intensity as a function of retinal location.

The first identifiable peak came at $325 \mathrm{msec}$, the next trough at $375 \mathrm{msec}$, and the following peak at $425 \mathrm{msec}$. A line joining the two peaks at 375 and $425 \mathrm{msec}$. was drawn. The signed perpendicular distance from the trough to this line was defined as the evoked potential amplitude measure. Figure 4 gives the plot of the average amplitude (based on the two observations) as a function of retinal location for each of the three stimulus intensities.

\section{DISCUSSION}

The blind spot can be detected using averaged EEG measures evoked with a small scotopic stimulus. The long latency of the onset of the evaked response was a function of the intensity of the stimulus. The low intensity of the stimulus was important in the 


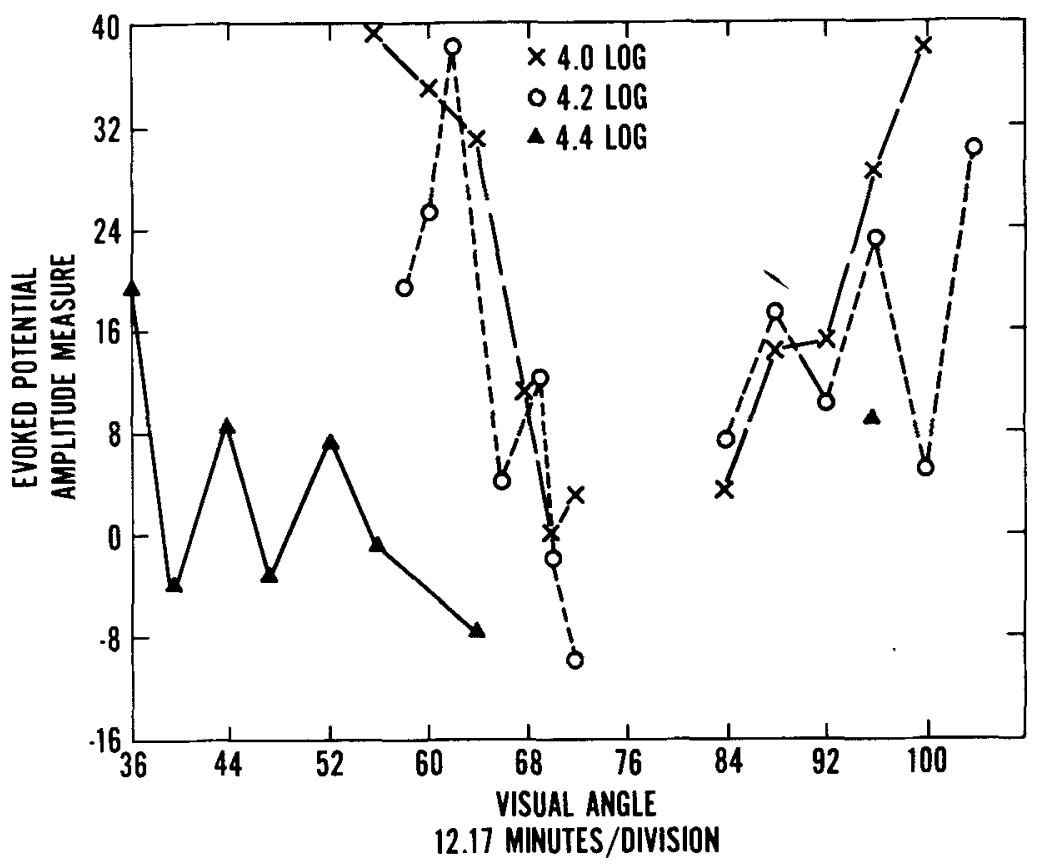

Fig. 4. Evoked potential amplitude measure as a function of retinal location for three stimulus intensities (4.0 log was brightest, 4.4 log was dimmest). Each point is based on 400 observations of a stimulus light. The abscissa scale can be compared with the scales in Figs. 2 and 3. electrical detection of the blind spot. Using only $\log 3.8$ neutral density Wratten filters the stimulus was seen across the entire blind spot measured by detection thresholds. This was likely caused by intraocular light scatter.

The selection of $\mathrm{S}$ is important. $\mathrm{S}$ must have an inherent low electrical noise level and remain attentive throughout the long recording sessions to insure that only minimum noise is mixed with the evoked response.

The sensitivity of the psychophysical and the electrophysiological measures compare favorably for the two highest stimulus intensities. However, each point on the average EEG curves was based on 400 observations of the stimulus while each point on the detection plots was based on at least 40 but not over 70 observations.

No averaged EEG evidence exists to support the hypothesis that $\mathrm{S}$ saw the light at the dimmest intensity. Below 56 on the scale $S$ had no detection errors. At this stimulus intensity detection thresholds were more sensitive.

At the present time it is concluded that a detection method provides a more sensitive and easily obtainable visual threshold measure than electroencephalographic methods.

\section{References}

Cobb, W. A. \& Dawson, G. O. The latency and form in man of the occipital potentials evoked by bright flashes. J. Physiol., 1960 , 152, 108-121.

Copenhaver, R. M., \& Beinhocker, G. D. Evoked occipital potentials recorded from scalp electrodes in response to focal visual illumination. Investigative Ophthal., 1963, 2, No. 4, 393-406.

Rietveld, W. J. The oddipitocortical response to light flashes in man. Acta Physiol. Pharmacol. Nearl., 1963, 12, 373-407.

Tepas, D. I., \& Armington, J. C. Properties of evoked visual potentials. Vision Res., 1962, 2, 449-461.

\section{Notes}

1. These data were presented orally at the Midwestern Psychological Assn. meeting in May 1966. The opinions and assertions contained herein are the private ones of the author.

2. This study was done while this author was a NAS-NRC postdoctoral research associate under the sponsorship of $\mathbf{C}$. $\mathbf{T}$. White and R. G. Eason, now at Washington State University.

(Accepted for publication December 9, 1966.) 